\title{
Composição química e potencial antioxidante de Jaracatia spinosa e Vasconcellea quercifolia A. St-Hil e o uso na alimentação: uma breve revisão
}

\author{
Chemical composition and antioxidant potential of Jaracatia spinosa and Vasconcellea quercifolia
}

\author{
A. St-Hil and their use in food: a brief review \\ Composición química y potencial antioxidante de Jaracatia spinosa y Vasconcellea quercifolia A. St-
}

Hil y su uso em alimentos: uma breve revisión

Recebido: 09/03/2021 | Revisado: 15/03/2021 | Aceito: 18/03/2021 | Publicado: 26/03/2021

\author{
Heloisa Dias Barbosa \\ ORCID: https://orcid.org/0000-0002-1220-3652 \\ Universidade Estadual de Maringá, Brasil \\ E-mail: heloisabbsa@gmail.com \\ Anderson Lazzari \\ ORCID: https://orcid.org/0000-0003-4039-4772 \\ Universidade Estadual de Maringá, Brasil \\ E-mail: anderson.lazzari29@gmail.com \\ Ingrid Caroline da Silva \\ ORCID: https://orcid.org/0000-0002-3606-0107 \\ Universidade Estadual de Maringá, Brasil \\ E-mail: ingrid_caroline95@hotmail.com \\ Lucas Henrique Maldonado da Silva \\ ORCID: https://orcid.org/0000-0002-8835-3868 \\ Universidade Estadual de Maringá, Paraná \\ E-mail: lucasmaldonado7@gmail.com \\ Ana Paula Dada \\ ORCID: https://orcid.org/0000-0002-4548-9183 \\ Universidade Estadual de Maringá, Brasil \\ E-mail: anapauladada2015@gmail.com \\ Andresa Caroline de Oliveira Cestário \\ ORCID: https://orcid.org/0000-0001-8524-006X \\ Universidade Estadual de Maringá, Brasil \\ E-mail: andresa_cestario@hotmail.com \\ Evandro Ribeiro Machado Filho \\ ORCID: https://orcid.org/0000-0003-4065-6074 \\ Universidade Estadual de Maringá, Brasil \\ E-mail: evandromachadofilho@gmail.com
}

\begin{abstract}
Resumo
O Brasil é reconhecido por sua riqueza em variedades de plantas e frutas nativas. Esta revisão tem como objetivo avaliar as pesquisas relacionadas quanto a plantas não convencionais e frutas exóticas, direcionados às frutas nativas do Brasil. Dentre elas, pouco se conhece sobre as frutas Jaracatia spinosa e Vasconcela quercifolia, conhecidas apenas regionalmente. Portanto, através de busca com os descritivos 'PANCS'; 'frutas exóticas brasileiras', 'Jaracatia spinosa', 'Vasconcella quercifolia' nas bases Scielo, Science direct e Web of Science foram selecionados os artigos que pudessem concentrar informações sobre sua composição nutricional e bioquímica, a fim de expandir o conhecimento sobre as propriedades das mesmas. Como por exemplo, com relação ao teor de fibra alimentar, para as duas frutas estudadas obteve-se resultados maiores do que para o mamão (Carica papaya) também pertencente à família Cariaceae, o que valoriza a importância de disseminar nacionalmente o consumo dessas outras frutas nativas e estimular seu consumo e aproveitamento.
\end{abstract}

Palavras-chave: Plantas comestíveis; Antioxidantes; Alimentos funcionais.

\begin{abstract}
Brazil is recognized for its wealth of varieties of native plants and fruits. This review aims to evaluate research related to non-conventional plants and exotic fruits, directed to native fruits of Brazil. Among them, little is known about the Jaracatia spinosa and Vasconcela quercifolia fruits, known only regionally. Therefore, by searching with the descriptive 'PANCs'; 'Brazilian exotic fruits', 'Jaracatia spinosa', 'Vasconcella quercifolia' in the Scielo, Science direct and Web of Science databases, articles that could concentrate information on their nutritional and biochemical composition were selected in order to expand knowledge about their properties. As for example, regarding dietary
\end{abstract}


fiber content, for the two fruits studied, greater results were obtained than for papaya (Carica papaya) also belonging to the Cariaceae family, which values the importance of spreading the consumption of these other fruits nationwide and encourage their consumption and use.

Keywords: Edible plants; Antioxidant; Functional food.

\section{Resumen}

Brasil es reconocido por su riqueza en variedades de plantas y frutas nativas. Esta revisión tiene como objetivo evaluar la investigación relacionada con plantas no convencionales y frutas exóticas, dirigida a frutas nativas de Brasil. Entre ellos, se sabe poco sobre las frutas Jaracatia spinosa y Vasconcela quercifolia, conocidas solo a nível regional. Por lo tanto, a través de la búsqueda con el descriptivo "PANCS"; 'Frutas exóticas brasileñas', 'Jaracatia spinosa', 'Vasconcella quercifolia' en las bases de datos Scielo, Science direct y Web of Science, se seleccionaron artículos que pudieran concentrar información sobre su composición nutricional y bioquímica, con el fin de ampliar el conocimiento sobre sus propiedades. Como por ejemplo, en cuanto al contenido de fibra dietética, se obtuvieron resultados superiores para las dos frutas estudiadas que para la papaya (Carica papaya) también perteneciente a la familia Cariaceae, que valora la importancia de difundir el consumo de estas otras frutas a nivel nacional y fomentar su consumo y uso.

Palabras clave: Plantas comestibles; Antioxidantes; Alimentos funcionales.

\section{Introdução}

O Brasil com $20 \%$ do número total de espécies na Terra, tem a maior biodiversidade do mundo. Embora a biodiversidade da flora brasileira seja amplamente conhecida, apenas uma pequena parte dela tem sido explorada, oferecendo oportunidades sem precedentes para a descoberta de tecidos vegetais comestíveis, ricos em nutrientes e compostos bioativos (Peisino et al., 2020). Dessa forma, surgem plantas consideradas ruderais, sendo conhecidas como plantas alimentícias não convencionais (PANCs). Essas plantas crescem espontaneamente e sem aditivos químicos, o consumo delas aumentou nas últimas décadas, o que pode estar diretamente ligado a estudos que mostram um maior valor nutricional, muitas vezes mais significativo em comparação com outras plantas alimentares comuns (Azam et al., 2014).

Com relação ao valor nutricional, pode-se destacar a família Caricaceae, a qual possui 30 espécies distribuídas em quatro gêneros: Cylicomorpha, Jaracatia, Carica e Jarilla. Conhecida principalmente pelo mamão (Carica papaya L.), possui uma diversidade de outras árvores e frutos que são conhecidos regionalmente e pouco conhecidos pelas suas características físicas, químicas e nutricionais (Badillo \& Leal, 2020).

Entre as potencias PANCs da família Caricaceae, pode-se destacar o Jaracatia spinosa e Vasconcellea quercifolia, ambas de origem brasileira. O Jaracatia spinosa é conhecido popularmente como mamoeiro-bravo, mamoeiro-do-mato e mamãozinho, é encontrado por todo o Brasil, em grande parte na região Sudeste. Seu fruto pode ser consumido in natura, em preparo de sucos e o cerne da árvore é comumente utilizado no preparo de doces. Mesmo com alguns exemplos de uso, ainda é uma fruta pouco conhecida e existe uma pouca informação e estudos sobre sua composição bioquímica e benefícios de seu consumo (Aguiar; Almeida; Carmargos., 2012).

Com ocorrência do sul da Bahia ao Rio Grande do Sul, Vasconcellea quercifolia A.St-Hil., popularmente conhecida como mamão do mato ou jaracatiá, é considerada uma Planta Alimentar Não Convencional (PANC) que possui poucos estudos sobre suas características nutricionais e funcionais. Sua comercialização ou de seus derivados é feita da forma in natura. Antigamente, em substituição ao coco no preparo de doces, o seu parênquima medular era ralado e utilizado (Folharini et al., 2019).

Portanto, foi feito um levantamento bibliográfico com o objetivo de reunir informações sobre as frutas 'Jaracatia spinosa' e 'Vasconcella quercifolia' visando ampliar o conhecimento sobre o potencial nutricional de consumo destas frutas. 


\section{Metodologia}

Realizou-se um levantamento bibliográfico na base de dados Scielo, Science Direct e Web of Science, através dos termos 'PANCS'; 'frutas exóticas brasileiras', 'Jaracatia spinosa', 'Vasconcella quercifolia'.

Os artigos que foram inclusos no presente estudo, foram estudos publicados nos idiomas português e inglês nos últimos 5 anos (2015 - 2020), com algumas exceções, por tratar-se de estudos relevantes sobre o tema, e por ser um tema com escassez de literatura. Artigos não disponíveis na íntegra, com repetição nas bases de dados, ou que não se enquadravam na delimitação de tempo citada acima, e que estavam fora do tema proposto, foram excluídos.

Para análise dos artigos resultantes das pesquisas, as palavras-chaves utilizadas foram "Plantas Alimentares NãoConvencionais", "Frutas exóticas", "Jaracatia spinosa" e "Vasconcella quercifolia", foram selecionados os que apresentavam em seu título/resumo, informação sobre plantas alimentícias não convencionais (PANCs), valor nutricional e compostos bioativos de frutas exóticas, em especial frutas da família Caricaceae, com o objetivo de explorar e valorizar a biodiversidade brasileira. Assim, denomina-se uma metodológica qualitativa em uma revisão bibliográfica (Pereira, Shitsuka, Pereira, \& Shitsuka, 2018).

\section{Resultados e Discussão}

Normalmente encontradas em calçadas, terrenos abandonados e até mesmo em monoculturas comercial, as plantas alimentícias não convencionais (PANCs) são hortaliças vivas, sendo muitas vezes consideradas como mato ou ervas daninhas, devido o seu fácil cultivo e proliferação (Biondo, Fleck, Kolchindi, Sant’anna \& Polesi, 2018).

Na Tabela 1, obtemos os resultados da pesquisa de artigos entre os anos de 2015 a 2020, publicações com os temas mais amplos, como "Plantas Alimentícias Não Convencionais" e "Frutas exóticas" são mais significativas, totalizando 2500 e 2435 respectivamente, sendo que a maior parte destas publicações estão relacionadas com o uso de PANCs e frutas exóticas na dieta humana. No entanto, quanto se refere às PANCs em específico, os resultados da pesquisa são bem menores.

Tabela 1. Número de artigos publicados nas bases de dados Science Direct, Scielo e Web of Science de acordo com as palavras-chave Plantas alimentícias Não-Convencionais, Frutas exóticas, Jaracatia spinosa e Vasconcella quercifolia.

\begin{tabular}{lccc}
\hline \multicolumn{1}{c}{ Palavras-chaves } & Science direct & Scielo & Web of science \\
\hline Plantas Alimentícias Não Convencionais & 2469 & 0 & 31 \\
Frutas exóticas & 2332 & 13 & 90 \\
Jacaratia spinosa & 10 & 2 & 31 \\
Vasconcella quercifolia & 1 & 2 & 8 \\
\hline
\end{tabular}

Fonte: Autoria própria (2020).

Em todas as bases de dados, os termos "Jaracatia spinosa" e "Vasconcella quercifolia", possuem poucos artigos publicados, isso mostra que pouco se sabe sobre essas Plantas Alimentícias Não-Convencionais, em relação ao seu consumo, composição química e compostos bioativos.

A fruta Jaracatiá tem origem no Cerrado brasileiro, mas está presente por toda a extensão do país, da Bahia até o Rio Grande do Sul. Em ponto de colheita, apresenta coloração alaranjada, normalmente consumida como suco ou utilizada em preparo de geleias (Aguiar et al., 2012; Negri, Berni \& Brazaca 2016). 
Em estudo para caracterização da fruta (polpa com a casca) da fruta tropical Jaracatiá foram obtidos valores de 82,4\% para umidade, $1,03 \%$ de proteína, $1,78 \%$ de cinzas, $0,24 \%$ de lipídeos, $0,45 \%$ de fibra alimentar solúvel, 4,50\% de fibra alimentar insolúvel e 9,63\% de carboidratos. Diferentes valores para fibra solúvel de 1,98 g/100g e de 4,70g/100g para fibras insolúveis foram relatados em outra pesquisa, sendo essa variação relacionada com a localização geográfica e estágio de amadurecimento da fruta (Berni, Campoli, Negri, Toledo \& Canniatti-Brazaca, 2019; Prospero, Da Silva \& Spoto, 2016). Consideradas também frutas tropicais, o teor de proteína é similar ao já encontrado para acerola $(1,08 \%)$ e maior do que para a pitanga $(0,62 \%)$. Para fibra alimentar total, a fruta Jaracatiá também apresentou maior resultado $(4,95 \%)$ do que acerola $(1,38 \%)$ e pitanga (2,99\%), possuindo assim efeitos fisiológicos relacionados ao seu consumo, uma vez que o consumo de fibras está relacionado com redução de doenças cardiovasculares e melhora do trânsito intestinal. Comparado com o mamão, da mesma família Caricaceae, o Jaracatiá in natura apresenta teor de minerais cerca de quase três vezes maior com relação ao cálcio e duas vezes maior com relação ao conteúdo de ferro (Berni et al., 2019; Prospero et al., 2016).

A composição de carotenoides da fruta também permitiu obter que os maiores compostos são: trans- $\beta \mathrm{C}$ com $18 \%$ de bioacessibilidade $\alpha$-C com valor entre 3 a $15 \%$ de bioacessibilidade. Além disso, no estudo de atividade de retinol equivalente, a fruta obteve 104,8 u/100 g o que sugere que seu consumo aumenta a ingestão de vitamina A (Berni et al., 2019).

Rocha et al. (2011) realizaram um estudo para determinar qual solvente teria melhor resultado na obtenção de fenólicos totais e taninos condensados. A extração foi mais eficiente com o etanol 95\%, obtendo $252 \pm 16 \mathrm{mg} / 100 \mathrm{~g}$ ácido gálico equivalente, comparado com a extração com acetona 70\% (105 \pm 4$)$ e metanol (135 \pm 13). Já para taninos, obteve maior resultado utilizando o solvente acetona $70 \% 79 \pm 4 \mathrm{mg}$ catequina equivalente/100g. O resultado para fenólicos totais foi maior para o Jaracatia do que para o mamão (Carica papaya L. var Formosa) que apresentou cerca de $88,1 \mathrm{mg} / 100 \mathrm{~g}$ ácido gálico, sendo considerada uma fruta fonte de compostos antioxidantes (Oliveira, Aquino, Ribeiro, Proença \& Pinheiro-Sant'Ana, 2011).

Além da avaliação do fruto de Jaracatia spinosa, o cerne da planta também costuma ser utilizado localmente, principalmente na produção de doces. Portanto, Aguiar et. al (2012) realizaram uma pesquisa para verificar os compostos presentes nesta parte do caule. De acordo com estes autores, ele é caracterizado por possuir cerca de 5,20 $\mu \mathrm{moles} / \mathrm{g}$ matéria fresca e 11,3620 $\mu$ moles/g matéria seca de aminoácidos solúveis totais. Além disso, 23,77\% da matéria seca é composta de açúcares solúveis totais, além de 7,1\% de açúcares redutores, 1,54\% de frutose e 10,64\% de sacarose. Também foi verificado que na medula caulinar há presença de 1,55g/100g de fibra solúvel e 6,45g/100g para fibra insolúvel (Prospero et. al,2016).

Considerada como uma Planta Alimentar Não Convencional e também com ocorrência do sul da Bahia ao Rio Grande do Sul, Vasconcellea quercifolia A. St-Hil possui poucos estudos sobre suas características nutricionais. Folharini et al. (2019) realizaram um estudo sobre a caracterização nutricional de Vasconcellea quercifolia em que os teores foram calculados em $\mathrm{g}$ por $100 \mathrm{~g}$ de amostra. O maior teor de proteínas totais foi encontrado no fruto verde $(2,09 \pm 0,55)$, o menor teor no parênquima medular $(0,58 \pm 0,05)$ e para o fruto amadurecido foi obtido $1,75 \pm 0,17$. Com relação ao teor de fibras, o maior teor encontrado também foi no fruto verde $(3,67 \pm 0,03)$ e menor no parênquima medular $(2,16 \pm 0,03)$ e quanto o teor de cinzas que é importante para a determinação dos minerais encontrados no fruto, obteve-se o maior teor no fruto verde $(2,09 \pm 0,55)$. O mamão papaya (Carica papaya Linn., da mesma família Caricaceae que é mais conhecido e consumido popularmente apresentou resultados menores para teores de proteína, fibra e minerais tanto do fruto verde $(0,7 \mathrm{~g} / 100 \mathrm{~g} ; 0,9 \mathrm{~g} / 100 \mathrm{~g}$ e $0,5 \mathrm{~g} / 100)$ quanto amadurecido $(0,6 \mathrm{~g} / 100 \mathrm{~g} ; 0,8 \mathrm{~g} / 100 \mathrm{~g}$ e $0,5 \mathrm{~g} / 100 \mathrm{~g})$ respectivamente, demonstrando o alto valor nutricional referente ao consumo de Vasconcellea quercifolia (Krishna; Paridhavi \& Patel, 2008).

Em estudo fitoquímico e atividade antioxidante dos frutos e folhas de Vasconcellea quercifolia, avaliou-se o extrato etanólico dos frutos e folhas em que os compostos fenólicos foram dados em mg/g equivalente de ácido gálico (EAG) e sua maior concentração foi encontrada nas folhas $41,35 \mathrm{mg} / \mathrm{g}$ EAG e para o fruto foi encontrado o valor de 19,47 mg/g EAG. Para 
flavonoides foram quantificados em $\mathrm{mg} / \mathrm{g}$ equivalente de quercetina (EQ), obtendo-se 7,33 mg/g EQ nas folhas e 0,60 mg/g quercetina para os frutos. A atividade antioxidante foi calculada pelo método DPPH e os valores expressos em IC50, o valor encontrado nas folhas foi de 669,10 $\mu \mathrm{g} / \mathrm{mL}$ (Leme, Vencato, Biondo, Boeira, \& Ferraz, 2017). A presença de compostos fitoquímicos, tais como flavonoides, carotenoides e bioativos possuem características antioxidantes, anti-inflamatórias, antimicrobianas e anticarcinogências, sendo benéfico o seu consumo e aumentando seu potencial funcional para consumo, visto que o mercado consumidor está atento e à procura de alimentos com funcionalidade e valor agregado à saúde (Jacob 2020, Bezerra \& De Brito, 2020).

Estudos sobre algumas Plantas Alimentares Não Convencionais estão presentes na literatura demonstrando que devido ao seu potencial nutritivo conseguiram ser introduzidas na alimentação humana (Garcia et al.,2017; Land, Borges, Borges \& Pascoal, 2017; Arellano-Acunã, Rojas-Zavaleta \& Paucar-Menacho, 2016; Silva, Azevedo \& Azevedo, 2015.

Em outro estudo, Negri \& Berni (2016) apresentaram oito plantas nativas que poderiam ser facilmente adicionadas à dieta popular, pela sua riqueza de nutrientes, minerais e compostos antioxidantes. Apesar de suas características nutricionais serem benéficas à saúde, elas são pouco aproveitas na alimentação pela escassez de informações.

\section{Conclusão}

Com base nos resultados explorados, Jaracatia spinosa e V. quercifolia, podem ser explorados como alimento, visando desenvolvimento agrícola, melhoramento econômico e como culturas de rotação, devido à sua adaptação às diversas condições ambientais e rápido crescimento. Elas apresentaram composições nutricionais maiores do que o mamão, sendo da mesma família Caricaceae além de também possuir compostos bioativos e antioxidantes, agregando propriedades funcionais com o seu consumo. É necessário maior difusão de informação, para permitir melhor consumo e maneiras de aproveitamento dessas frutas e assim integrarem não somente o mercado regional, mas atingir o consumo e conhecimento nacional.

\section{Agradecimentos}

Os autores agradecem a Coordenação de Aperfeiçoamento de Pessoal Superior (CAPES) pela assistência financeira.

\section{Referências}

Aguiar, L. F., Almeida, C. A., \& Camargos, L. S. (2012). Biochemistry characterization of the stem composition of the Jaracatia (Jaracatia spinosa). Acta Iguazu, 1, 65-71.

Arellano-Acuña, E., Rojas-Zavaleta, I., \& Paucar-Menacho, L. M. (2016). Fruta tropical de excelentes propiedades funcionales que ayudan a mejorar la calidad de vida. Scientia Agropecuaria, 7, 433-443.

Azam, F. M. S., Biswas, A., Mannan, A., Afsana, N. A., Jahan, R., \& Rahmatullah, M. (2014). Are famine food plants also ethnomedicinal plants? An ethnomedicinal appraisal of famine food plants of two districts of Bangladesh. Evid. Based Complement. Altern. Med., 2014, 1-28.

Badillo, V. M., \& Leal, F. (2020). Taxonomy and Botany of the Caricaceae.

Berni, P., Campoli, S. S., Negri, T. C., Toledo, N. M. V., \& Canniatti-Brazaca, S. G. C. (2019). Non-conventional Tropical Fruits: Characterization, Antioxidant Potential, and carotenoid bioaccessibility. Plant Foods for Human Nutrition, 74, 141-148.

Bezerra, J. A., De Brito, M. M. (2020). Potencial nutricional e antioxidantes das plantas alimentícias não convencionais (PANCs) e o uso na alimentação: Revisão. Research, Society and Development, 9 (9), e369997159.

Biondo, E., Fleck, M., Kolchindki, E. M., Sant'anna, V., \& Polesi, R. G. (2018). Diversidade e potencial de utilização de plantas alimentícias não convencionais ocorrentes no Vale do Taquari, RS. Revista Eletrônica de Ciências da UERGS, 4,61-90.

Folharini, Z. F., Orlandi, C. R., Martini, M. C., Bruxel, F., Altamayer, T., Brietzke, D. T., Gonçalves, T. E., Finatto, J., Ethur, E. M., Moura, N. F., Hoehne, L., \& Freitas, E. M. (2019). Nutritional characterization of Vasconcellea quercifolia A.St-Hil.: potential for the development of functional food. Food Sci. Technol., 39, 432-438.

Garcia, L. G. C., Guimarães, W. F., Rodovalho, E. C., Peres, N. R. A. A., Becker, F. S., \& Damiani, C. (2017). Geleia de buriti (Mauritia flexuosa): agregação de valor aos frutos do cerrado brasileiro. Brazilian Journal of Food Technology, 20, 1-5. 
Research, Society and Development, v. 10, n. 3, e54810313709, 2021

(CC BY 4.0) | ISSN 2525-3409 | DOI: http://dx.doi.org/10.33448/rsd-v10i3.13709

Jacob, M. C. M. (2020). Biodiversidade de plantas alimentícias não convencionais em uma horta comunitária com fins educativos. Demetra, 15 , 1-17.

Krishna, K. L., Paridhavi, M. \& Patel, J. A. (2008). Review on nutritional, medicinal and pharmacological properties of Papaya (Carica papaya Linn.). Natural product radiance, 7 (4), 364-373.

Land, L. R. B., Borges, F. M., Borges, D. O., \& Pascoal, G. B. (2017). Composição centesimal, compostos bioativos e parâmetros físico-químicos da mamacadela (Brosimum gaudichaudii Tréc) proveniente do Cerrado Mineiro. Demetra. 12, 509-518.

Leme, M. L. B., Vencato, S. B., Biondo, E., Boeira, J., \& Ferraz, A. B. F. (2017). Análise fitoquímica de antioxidante dos frutos e folhas de Vasconcellea quercifolia. In: Expo Ulbra, 2017, Canoas. Anais do XXII Salão de Iniciação Científica e Tecnológica.

Negri, T. C., Berni, P. R. A., \& Brazaca, S. G. C. (2016). Valor nutricional de frutas nativas e exóticas do Brasil. Biosaúde, 18, 82-96.

Oliveira, D. S., Aquino, P. P., Ribeiro, S. M., Proença, R. P. C. \& Pinheiro-Sant'Ana, H. M. (2011). Acta Scientiarum Health Sciences, 33 (1), 89-98.

Peisino, M. C. O., Zouain, M. S., Scherer, M. M. C., Schmitt, P., Silva, M. V. T., Barth, T., Endringer, D. C., Scherer, R., \& Fronza, M. (2020). HealthPromoting Properties of Brazilian Unconventional Food Plants. Waste and Biomass Valorization, 11, 4691-4700.

Pereira, A. S., Shitsuka, D. M., Pereira, F. J., \& Shitsuka, R. (2018). Metodologia da pesquisa científica. UFSM. https://repositorio.ufsm.br/bitstream/handle/ 1/15824/Lic_Computacao_Metodologia-Pesquisa-Cientifica.pdf?sequence=1.

Prospero, E. T.; Da Silva, P. P. \& Spoto, M. H. (2016). Caracterização físico-química, nutricional e de compostos voláteis de frutos de Jaracatia spinosa provenientes de três regiões do estado de São Paulo - Brasil. Revista Brasileira de Tecnologia Agroindustrial, 10 (1), $2095-2111$.

Rocha, W. S., Lopes, R. M., Da Silva, D. B., Vieira, R. F., Da Silva, J. P., \& Agostini-Costa, T. S. (2011). Compostos fenólicos totais e taninos condensados em frutas nativas do cerrado. Revista Brasileira de Fruticultura, 33, 1215-1221.

Silva, B. L. A., Azevedo, C. C., \& Azevedo, F. L. A. A. (2015). Propriedades funcionais das proteínas de amêndoas da munguba (Pachira aquática Aubl.). Revista Brasileira de Fruticultura, 37, 193-200. 